\section{A survey of severe visual impairment in children attending schools for the blind in a coastal district of Andhra Pradesh in South India}

\begin{abstract}
Purpose To identify the major causes of severe childhood visual impairment and blindness among students attending schools for the blind in a coastal district of Andhra Pradesh (AP) in South India.

Methods Children $\leq 16$ years of age attending six schools for the blind in the study area were interviewed and examined in the year 2009, and causes were classified according to the World Health Organization Program for Prevention of Blindness (WHO/ PBL) childhood blindness proforma. A total of 113 children underwent a detailed eye examination by an experienced ophthalmologist.

Results The major causes of blindness were congenital eye anomalies in 46 children (41.4; 95\% confidence interval (CI): 32.3-50.6), followed by retinal disorders in 21 children (18.9\%; 95\% CI: 11.6-26.2), cataract in 9 children (9.7\%; 95\% CI: 2.9-12.9), and corneal conditions (scar and Staphyloma) in 8 children (7.1\%; 95\% CI: 2.4-11.8). More than half the children $(56.6 \%)$ were blind due to conditions that could have been treated or prevented. Discussion Congenital anomalies were found to be the most common cause of blindness. The majority of the cases were due to avoidable causes of blindness. Therefore, robust screening measures may help reduce the burden of visual impairment in children. Eye (2012) 26, 1065-1070; doi:10.1038/eye.2012.88; published online 11 May 2012
\end{abstract}

Keywords: avoidable childhood blindness; schools for the blind; coastal Andhra Pradesh; congenital eye diseases; hereditary blindness
S Krishnaiah ${ }^{1,2}$, B Subba Rao ${ }^{3,4}$,

K Lakshmi Narasamma ${ }^{5}$ and $G$ Amit $^{6}$

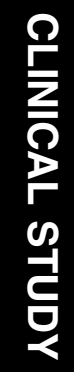

${ }^{1}$ Centre for Clinical

Epidemiology and

Biostatistics, L V Prasad Eye Institute, Kallam Anji Reddy

Campus, Hyderabad, India

${ }^{2}$ International Centre for Advancement of Rural Eye Care, L V Prasad Eye Institute, Hyderabad, India

${ }^{3}$ Community Health and Nutrition Office, Prathipadu, India

${ }^{4}$ School of Optometry and Vision Science, University of New South Wales, Sydney, New South Wales, Australia

${ }^{5}$ Lakshmi Eye Hospital, East Godavari, India

${ }^{6} J a s t i$ V Ramanamma Children's Eye Care Centre, L V Prasad Eye Institute, Hyderabad, India

Correspondence: $S$ Krishnaiah, Centre for Clinical Epidemiology and Biostatistics, L V Prasad Eye Institute, Kallam Anji Reddy Campus, Banjara Hills, Hyderabad 500034, Andhra Pradesh, India

Tel: + 91403061 5602; Fax: + 914023548271

E-mail: krishnaiah@lvpei.org or skrishna_s@yahoo.com

Received: 6 January 2012 Accepted in revised form: 28 March 2012

Published online: 11 May 2012

This manuscript submission represents original research that has not been published previously. This manuscript has not been submitted for consideration elsewhere for publication and does not duplicate the material published already. childhood visual impairment and blindness. A study conducted in Delhi in which children were examined reported that $92.9 \%$ of children were blind (presenting visual acuity (VA) in better eye $<3 / 60) .{ }^{7}$ Another survey conducted in Maharashtra in which children $<16$ years of age from 35 residential schools for the blind were examined reported that $95.4 \%$ of children were blind (presenting VA in better eye $<3 / 60$ ). ${ }^{8}$

In order to set priorities for control programmes related to childhood blindness in 
India, epidemiological data of the prevalence and major causes of childhood blindness is required from different sections of the population. Reliable population-based data on the causes of blindness in children is difficult to obtain. One way to obtain data on blind children is doing surveys of visual impairment and blindness in children attending schools for the blind. Over the past few years, usage of the World Health Organization's classification system has been increasing that allows for data to be compared across studies. ${ }^{9}$

The present study was conducted in East Godavari district, which is one of the coastal districts of Andhra Pradesh, a prosperous, well-irrigated region in the state. The primary objective of this study was to determine the causes of blindness in children attending the schools for the blind in this district.

\section{Materials and methods}

Six schools for the blind were identified in the district as part of this study. These schools were located in Kakinada, the district headquarters and in Rajahmundry, Rajavolu, Mandapeta, Ramachandrapuram and Amalapuram. The District Collector was informed about this study; all schools were visited and prior permission was obtained from the respective Principals of the various schools to conduct the study. Approval from the Ethics Committee was obtained from the Institutional Review Board of L V Prasad Eye Institute, Hyderabad, and the study was conducted during June and September 2009 following the tenets of the Helsinki Declaration.

Relevant information was collected from the teachers and parents (wherever possible) by reviewing school medical records and by administering a semi-open ended questionnaire to the children's parents. Brief information on demographics of age, gender, and place of residence were collected. A brief history of the family, age of onset of visual loss, and whether the parent's marriage was consanguineous was documented. Information on additional disabilities such as hearing loss, mental retardation, physical handicap, and epilepsy was also obtained from the children's records. Detailed eye examination was performed by a team comprising an ophthalmologist, and two ophthalmic officers who are mid-level ophthalmic personnel trained in vision screening and refraction. Distance VA was measured using a Snellen E chart. The VA was tested separately for each eye and then for both eyes together. The assessment of functional vision was performed for both the eyes together. Functional vision was assessed to determine if the child had useful residual vision for independent mobility (the ability to navigate without assistance between two chairs set $2 \mathrm{~m}$ apart in a well-lit room), social contact (the ability to recognize known faces at a distance of $2 \mathrm{~m}$ ), and near vision (the ability to recognize or describe the shape of three $5 \mathrm{~mm}$ symbols at any near distance).$^{10}$ Assessment for refraction and low vision aids was performed in all children who were able to perform the tests for functional vision. Anterior segment examination was performed using a torch, magnifying loupe, and/or slit lamp. The posterior segment examination was done after dilating the pupil, where indicated, using a direct and/or indirect ophthalmoscope.

The standard World Health Organization Program for Prevention of Blindness (WHO/PBL) eye examination record for children with blindness and low vision protocol was used to categorize the causes of blindness and to record findings, using the definitions in the coding instruments. ${ }^{9}$ A major anatomical site and underlying cause was selected for each eye, and for each child. For each child, the need for optical, surgical, or medical interventions and low vision aids was recorded and the expected visual prognosis assessed. Children requiring further investigations and treatment were referred to the district hospital for further management. The data was analyzed using SPSS 17.0 version for Windows (SPSS, Chicago, IL, USA).

\section{Results}

A total of $113(95.0 \%)$ children out of the 119 students attending six schools in the district were examined. There were six students $>16$ years old who were excluded from the study. A total of $63(55.8 \%)$ children examined were male. The mean age of the children was $10.8 \pm 2.9$ and the age ranged from 4 to 15 years. A history of consanguineous marriage of the parents was recorded in half of the children examined, $48.7 \%$ (95\% CI: 39.5-57.9; $n=55)$ in this study.

\section{Categories of visual loss}

Levels of VA for the 113 children attending these schools for the blind, in accordance with the WHO criteria for category of vision loss are shown in Table 1. Only two children (1.8\%) had vision impairment (best corrected VA

Table 1 Visual impairment and blindness status in 113 children by WHO categories

\begin{tabular}{lccc}
\hline Visual acuity & WHO categories & No. & $\%(95 \%$ CI) \\
\hline $6 / 6-6 / 18$ & NI & 0 & $0(0)$ \\
$<6 / 18-6 / 60$ & VI & 2 & $1.8(0-4.2)$ \\
$<6 / 60-3 / 60$ & SVI & 8 & $7.1(2.4-11.8)$ \\
$<3 / 60-$ PL & BL & 54 & $47.8(38.6-57.0)$ \\
NPL & BL & 49 & $43.4(34.2-52.5)$ \\
\hline
\end{tabular}

Abbreviations: BL, blindness; CI, confidence interval; NI, no impairment; SVI, severe visual impairment; VI, visual impairment. 
$<6 / 18$ to $6 / 60)$ and eight $(7.1 \%)$ children had severe visual impairment (VA $<6 / 60$ to $3 / 60$ ). Among the blind children, $54(47.8 \%)$ had VA in the range of less than $3 / 60$ to light perception, whereas $49(43.4 \%)$ had no light perception.

\section{Anatomical causes of severe visual impairment and blindness}

In the 111 children who had severe visual impairment or blindness, anophthalmos, microphthalmos, and phthisis were the most frequent globe abnormalities leading to visual loss, accounting for $41.4 \%$ of cases (Table 2 ). Retina-related problems were the second most frequent and were responsible for $18.9 \%$ of cases. Of the 21 children with retinal problems, 19 had dystrophy.

Table 2 Anatomical site of severe visual impairment and blindness in 111 children examined in six schools for blind in the study area

\begin{tabular}{|c|c|c|c|c|}
\hline Anatomical site & No. & No. & $\%$ & $\%$ \\
\hline Whole globe & 46 & & 41.4 & \\
\hline Anophthalmos & & 18 & & 16.2 \\
\hline Microphthalmos & & 24 & & 21.6 \\
\hline Phthisis & & 4 & & 3.6 \\
\hline Cornea & 9 & & 8.1 & \\
\hline Staphyloma & & 3 & & 2.7 \\
\hline Scar & & 5 & & 4.5 \\
\hline Dystrophy & & 1 & & 0.9 \\
\hline Lens & 11 & & 9.9 & \\
\hline Congenital cataract & & 9 & & 8.1 \\
\hline $\begin{array}{l}\text { Congenital ectopia lentis/ } \\
\text { micro spherophakia }\end{array}$ & & 2 & & 1.8 \\
\hline Uvea & 5 & & 4.5 & \\
\hline Uveitis & & 5 & & 4.5 \\
\hline Retina & 21 & & 18.9 & \\
\hline Dystrophy & & 19 & & 17.1 \\
\hline Albinism & & 1 & & 0.9 \\
\hline Retinoblastoma & & 1 & & 0.9 \\
\hline Optic nerve & 7 & & 6.3 & \\
\hline Atrophy & & 2 & & 1.8 \\
\hline Glaucoma & & 9 & & 8.1 \\
\hline Hypoplasia & & 3 & & 2.7 \\
\hline Coloboma of fundus & & 1 & & 0.9 \\
\hline Other causes & 12 & & 10.8 & \\
\hline Amblyopia & & 2 & & 1.8 \\
\hline Idiopathic nystagmus & & 2 & & 1.8 \\
\hline Total & 111 & 111 & 100 & 100 \\
\hline
\end{tabular}

\section{Aetiological categories of severe visual impairment and blindness}

Aetiological categories of severe visual impairment and blindness in these children are shown in Table 3.

Abnormality since birth (55\%) followed by hereditary diseases $(17.1 \%)$ were responsible for the majority of cases. Congenital rubella was responsible in only $0.9 \%$ of cases in this study.

\section{Avoidable causes of severe visual impairment and blindness}

In this study, $28.3 \%$ of children had lost vision as a result of avoidable causes. The main preventable causes (12.6\%) were trauma, ophthalmia neonatorum, and vitamin A deficiency (Table 4). An additional 15.9\% were blind from causes that were potentially treatable. Ophthalmia neonatorum was the major preventable cause of visual loss in five children and glaucoma and/ or buphthalmos in nine children. Uveitis and cataract, the other treatable causes of visual loss, were observed in five and three children, respectively.

Table 3 Aetiological classification based on the time of onset of visual loss of severe visual impairment and blindness in 111 children examined in six schools for blind in the study area

\begin{tabular}{lcccr}
\hline Aetiological cause & No. & No. & $\%$ & $\%$ \\
\hline Hereditary factors & 19 & & 17.1 & \\
$\quad$ Autosomal recessive & & 5 & & 4.5 \\
$\quad$ Chromosomal abnormality & & 2 & & 1.8 \\
$\quad$ Cannot specify & & 12 & & 10.9 \\
$\quad$ Intrauterine factor & 1 & & 0.9 & \\
$\quad$ Rubella & & 1 & & 0.9
\end{tabular}

Perinatal/neonatal factors

Cerebral hypoxia/injury

Ophthalmia neonatorum

6

5.4

Postnatal/childhood factors

Vitamin A deficiency

Measles

Trauma

Others

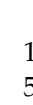

1
5

Undetermined

Cataract

Glaucoma

Retinoblastoma

Abnormality since birth

Others

9

8.1

0.9

0.9

3.6

2.7

Total

$\begin{array}{llll}111 & 111 & 100 & 100\end{array}$


Table 4 Avoidable causes of severe visual impairment/ blindness in children examined in six schools for blind in the study area

\begin{tabular}{lcc}
\hline Causes & Number & Percentage \\
\hline Preventable conditions & & \\
Vitamin A deficiency and Measles & 2 & 1.8 \\
Ametropic Amblyopia & 1 & 0.9 \\
Rubella & 1 & 0.9 \\
Stimulus deprivation Amblyopia & 1 & 0.9 \\
Trauma & 4 & 3.5 \\
Ophthamia neonatorum & 5 & 4.4 \\
Total preventable & 14 & 12.6 \\
& & \\
Treatable conditions & & \\
Cataract & 3 & 2.7 \\
Glaucoma & 9 & 8.0 \\
Uveitis & 5 & 4.4 \\
Pseudophakia with dense & 1 & 0.9 \\
Total treatable & 18 & 16.2 \\
& & \\
Total avoidable & 32 & 28.3 \\
\hline
\end{tabular}

\section{Discussion}

In order to develop control programs to prevent childhood blindness in the country, it is necessary to identify important avoidable causes of severe visual impairment and blindness, and monitor changing patterns from time to time. The study findings of major causes of blindness in children would be useful for the District Blindness Control Society (DBCS) in planning for control of blindness in children in the area of the studied population. There is a paucity of data available on childhood blindness in India. Population-based studies are not possible; therefore, the only option left was to study the schools for the blind. However, studies conducted on children studying in these special schools have limitations, as the children attending the schools may be different from blind children not attending school, and may not be representative of blind children in the whole population. In particular, for causes associated with additional disabilities, the children affected from poor communities are likely to be underrepresented due to high mortality arising from malnutrition along with additional disabilities and blindness. This is evident from earlier reports that in poor countries $60-80 \%$ of blind children die within $1-2$ years of becoming blind. ${ }^{11}$ Earlier, it was understood that in most developing countries only about $10 \%$ of blind children are in schools for the blind. ${ }^{12}$ Despite these limitations, data studied from such sources on the causes of childhood blindness do give valuable information. However, earlier it was inferred that the findings obtained from studies on special schools and community-based sample studies are comparable. ${ }^{13}$
This study showed that congenital ocular anomalies (mainly microphthalmos, anophthalmos, and phthisis) accounted for $41.4 \%$ of severe visual impairment and blindness (SVI/BL) in this coastal district population of AP. It can be speculated that congenital anomalies may be due to a genetic disorder, especially due to the fact that the majority of children were born to couples with a history of consanguineous marriage, which is common in this population. One hypothesis has been that microphthalmos, anophthalmos, and coloboma are due to interactions between genes controlling retinoic acid signalling and maternal vitamin A deficiency during early fetal development, a mechanism similar to folate deficiency and spina bifida with supportive evidence coming from animal experiments and epidemiological, and laboratory studies. The finding of higher rates of microphthalmos and anophthalmos has been found to be a common finding in many studies conducted earlier on blind children in India. 3,4,7,8,14,15 Congenital ocular anomalies were responsible for major causes of blindness in $41.3 \%$ and $27.4 \%$ of children in Maharashtra and Delhi, respectively. Retinal blindness was the second most common cause of SVI/BL $(18.9 \% ; n=21)$ identified in this population. The major cause was retinal dystrophy $(17.1 \% ; n=19)$, the finding approximately close to the estimate reported previously from the same population. ${ }^{14}$

This study found a low rate of corneal disease $(8.1 \%)$ in the children studied. A possible reason for a low rate of corneal disease observed in this study might be related to the higher coverage of immunization. The results of this study showed a mixed picture of causes with a predominance of hereditary and unknown aetiologies, a pattern similar to that in developed countries. A previously conducted study in India reported that the major cause of SVI/BL in children in schools for the blind was vitamin A deficiency. ${ }^{16}$ However, in this study only $0.9 \%$ of SVI/BL in children was attributed to vitamin A deficiency and was not a major cause of SVI/BL. This study also found a low rate of rubella $(0.9 \%)$ in the studied children, which certainly reflects improved primary healthcare, including greater rubella immunization coverage in private hospitals with close to $50 \%$ increased acceptance of immunization in the coastal districts of AP (Personal communication: Private Eye Health Hospitals, Kakinada, Andhra Pradesh).

In this study, $28.3 \%$ of children were found to have avoidable causes of vision loss. This finding is relatively less in magnitude when compared to the recent finding from Maharashtra. ${ }^{8}$ No child in our study was found to be blind due to retinopathy of prematurity (ROP). Though, this finding concurs with a recently published report from Maharashtra, ${ }^{8}$ however, ROP is found to be reaching epidemic proportions and becoming a public 
health problem not only in India but also in many middle income countries. ${ }^{17}$ Perhaps, ROP might not have been picked up in our current study due to these children being followed up appropriately at tertiary care centres for management. Though specialized neonatal care is unavailable currently at the district level primary care facility, it is a more focused activity at the tertiary care level, which could be one of the reasons that ROP was not found in the children comprising our study sample. Another possible reason could be the lack of survival of these children who were born at 28 weeks gestational age in these rural areas because of lack of availability of quality neonatal care.

Treatable causes included cataract, glaucoma, and uveitis. Congenital cataract is the leading cause of surgically correctable blindness in most developing countries. ${ }^{18}$ However, congenital cataract was not seen as a major cause of blindness in this study. In this study, there were nine children found with unoperated cataract of which six children had no perception of light and the remaining children with operable cataract were referred for surgery to the nearest tertiary care hospital. Early detection and treatment might have accounted for this reduction in this population. Alternatively, the selection of schools for the blind for the survey might have underestimated this observation. Whereas congenital glaucoma as a cause of SVI/BL was responsible in $8.1 \%$ of children in this study, which is approximately twice compared to the finding from North India. ${ }^{7}$ This is mainly because of high rates of consanguineous marriages in this part of the country. Despite the adverse effects caused by consanguineous unions, such relationships still occur in many countries. Previous studies have reported that $\sim 20-50 \%$ of marriages in central and southern Asia are usually consanguineous and it was $10-50 \%$ especially in South India. ${ }^{19}$ The high rates of such marriages in South India is mainly because consanguinity is a deeply rooted cultural tradition in this population. Families perceive premarital agreement regarding financial matters easier to conduct if the couple is related as cousins, as the marriage would consolidate and strengthen family ties. Besides the common perception in favour of consanguineous marriages, there is also a lack of awareness about the definite risks and possibilities for prevention of genetic disorders that is related to consanguinity. A few previous studies conducted in South India have also reported a very high prevalence of consanguinity (36\%) and a very low level of awareness $(7.6 \%)$, with regard to the hazards of consanguineous marriages. ${ }^{20}$ Hence, there is a need for screening, especially in families with consanguineous marriages, for early detection of glaucoma and cataract, with advocacy for appropriate referral to a tertiary care centre. Education and counselling on the effects of consanguineous marriages in primary health care settings will also help to address the problem. Therefore, counselling for couples with affected children, by giving reproductive options such as prenatal diagnosiswherever feasible-is especially beneficial. In addition, increasing awareness and educating the community about the problems of marrying close relatives, through appropriate and current government programs such as Accredited Social Health Activist and Integrated Child Development Scheme may help in addressing the problem in the long term.

The potential limitation of the study is that the prevalence of visual impairment and blindness might have been underestimated because this is not a population-based study. Designing and carrying out population-based studies to find prevalence of childhood blindness is challenging, as large sample sizes are required due to the very low prevalence of blindness in children. As far as blindness in children is concerned, sample sizes close to 20000-30000 children would be needed to provide precise estimates of the prevalence and the sample could need to be increased even further to obtain meaningful data on causes. In addition to this, taking measurements of VA in young children in a field setting can be extremely difficult. Preschool children, children with multiple disabilities, those living in tribal areas, and those who have died were likely to be underrepresented in this study. Therefore, the findings of this study need to be interpreted with great caution. This study, like any other studies in schools for the blind, is subject to selection bias even though it offered us the opportunity to examine many blind children within a short time. Another limitation of the study is that the report is based on data from a district in coastal Andhra Pradesh and the results might be relevant to the entire geographical area of coastal Andhra Pradesh only, but not for the other two regions, that is, Telangana and Rayalaseema in the state, as the latter regions are relatively less developed when compared to coastal Andhra Pradesh.

In conclusion, the pattern of childhood blindness seen in this study was comparable to the studies reported from North India. Close to $30 \%$ of the parents of the blind children had not approached eye care centre owing to lack of awareness, and $46 \%$ of parents did not approach paediatric eye care centres due to lack of availability. Despite rapid urbanization and increased rates of literacy in this area, there are $48.7 \%$ cases related to consanguineous marriages in this study that indicates the need for premarital counselling to avoid congenital ophthalmic disorders. Preventable causes of blindness can be reduced at the primary level of service delivery through measures such as improved vaccination coverage, including strengthening of the vitamin A 
supplementation programmes that are run by the government. Whereas treatable causes require specialized paediatric ophthalmology units and need a comprehensive approach in which systems are required for early identification and referral, including provision of low vision aids to children.

\section{Summary}

What was known before

- Childhood blindness: Childhood blindness from schools for the blind were studied extensively from north part of India.

- Blind school studies: However, very few studies have reported on causes of childhood blindness from the schools for the blind, which have reported previously.

What this study adds

- This study has provided different causes of childhood blindness from Southern part of India including the causes of childhood blindness as a result of consanguineous marriages that are high and common in this part of studied population.

- In addition, this study adds few discussions on the rates of consanguineous marriages and its associated data on awareness levels. Also few recommendations were made to increase the awareness on complications as a result of such consanguineous marriages.

\section{Conflict of interest}

The authors declare no conflict of interest.

\section{Acknowledgements}

We are thankful to Sethu Sheela Devi and Jatla Narsing Rao for administrative services; Sabera Banu for library services; Sreedevi Yadavalli for proof reading the manuscript and all children including their parents for participation in the study. This study was supported by the Hyderabad Eye Research Foundation, Hyderabad, India.

\section{References}

1 World Health organization. Global Initiative for the Elimination of Avoidable Blindness, WHO Publication Number WHO/PBL/97 61. Geneva:WHO, 1997.

2 World Health Organization. Fact Sheet No 2822009.

3 Rahi JS, Gilbert CE, Foster A, Minassian D. Measuring the burden of childhood blindness. Br J Ophthalmol 1999; 83: 387-388.
4 Dandona L, Williams JD, Williams BC, Rao GN. Population based assessment of childhood blindness in southern India. Arch Ophthalmol 1998; 116: 545-546.

5 Dandona R, Dandona L, Srinivas M, Sahare P, Narsaiah S, Muñoz SR et al. Refractive errors in children in a rural population in India. Invest Ophthalmol Vis Sci 2002; 43: 615-622.

6 Murthy GVS, Gupta SK, Elloveia LB, Muñoz SR, Pokharel GP, Sanga L et al. Refractive errors in children in an urban population in New Delhi. Invest Ophthalmol Vis Sci 2002; 43: 623-631.

7 Titiyal JS, Pal N, Murthy GVS, Gupta SK, Tandon R, Vajpayee RB et al. Causes and temporal trends of blindness and severe visual impairment in children in schools for the blind in North India. Br J Ophthalmol 2003; 87: 941-945.

8 Gogate P, Deshpande M, Sudrik S, Taras S, Kishore H, Gilbert $\mathrm{C}$ et al. Changing pattern of childhood blindness in Maharashtra, India. Br J Ophthalmol 2007; 91: 8-12.

9 Gilbert C, Foster A, Negrel AD, Thylefors B. Childhood blindness: a new form of recording causes of vision loss in children. Bull World Health Organ 1993; 71: 485-489.

10 Silver J, Gilbert CE, Spoerer P, Foster A. Low vision in east African blind school students: need for optical low vision services. Br J Ophthalmol 1995; 79: 814-820.

11 Lewallen S. Courtright. Blindness in Africa: present situation and future needs. Br J Ophthalmol 2001; 85: 897-903.

12 Gilbert C, Foster A. Childhood blindness in the context of Vision 2020 - the right to sight. Bull World Health Organ 2001; 79: 227-232.

13 Bulgan T, Gilbert C. Prevalence and causes of severe visual impairment and blindness in children in Mongolia. Ophthalmic Epidemiol 2002; 9: 271-281.

14 Hornby SJ, Adolph S, Gothwal VK, Gilbert CE, Dandona L, Foster A. Evaluation of children in six blind schools in Andhra Pradesh. Ind J Ophthalmol 2000; 48: 195-200.

15 Sil AK, Gilbert CE. Childhood blindness in India. J Ind Med Assoc 2001; 99: 10-15.

16 Rahi JS, Sripathi S, Gilbert CE, Foster A. Childhood blindness in India: causes in 1318 blind school students in nine states. Eye 1995; 9: 545-550.

17 Gilbert C, Fielder A, Gordillo L, Quinn G, Semiglia R, Visintin $\mathrm{P}$ et al. Characteristics of babies with severe retinopathy of prematurity in countries with low, moderate and high levels of development: implications for screening programmes. Pediatrics Electronic Pages 2005; 115: 518-525.

18 Rogers NK, Gilbert CE, Foster A, Foster A, Zakhidov BO, McCollum CJ. Childhood blindness in Uzbekistan. Eye 1995; 13: 65-70.

19 Yunis K, Rafei RE, Mumtaz G. Consanguinity perinatal outcomes and prevention - A view from the Middle East. Neoreviews 2008; 9: e59-e65.

20 Nath A, Patil C, Naik VA. Prevalence of consanguineous marriages in a rural community and its effects on pregnancy outcome. Indian J Com Med 2004; 29(1): 41-43. 